\title{
La participación ciudadana y las acciones comunitarias en la prevención del delito. Caso Nuevo León ${ }^{1}$ \\ Citizen participation and community actions in crime prevention. Case Nuevo Leon.
}

\author{
Dave Alexander López Mejía ${ }^{2}$ \\ iD http://orcid.org/0000-0003-3576-7749 \\ Juan de Dios Martínez Villarreal ${ }^{3}$ \\ iD http://orcid.org/0000-0002-5868-3786 \\ Universidad Autónoma de Nuevo León, México
}

\section{Como referenciar este artículo:}

López Mejía, D., A. \& Martínez Villareal, J., D. (2020) La participación ciudadana y las acciones comunitarias en la prevención del delito. Caso Nuevo León. Revista Ciencia Jurídica y Política, 80-103. Recuperado de: https://portalderevistas.upoli.edu.ni/index.php/5revciencasjuridicasypoliticas/article/view/637

\section{RESUMEN}

La prevención del delito es la herramienta adecuada y principal para la disminución de los índices de criminalidad en cualquier sociedad. En este artículo se hace una revisión sobre los modelos de participación ciudadana y las leyes vinculantes con la sociedad específicamente en materia de inseguridad, prevención del delito, mecanismos para la prevención, con los objetivos de determinar el grado de conocimiento de la población con respecto a la Ley de Prevención Social de la Violencia y la Delincuencia con Participación Ciudadana del Estado de Nuevo León y los niveles de participación ciudadana en la prevención del delito e identificar si las acciones sociales y gubernamentales han sido eficientes en la prevención del delito; en lo que corresponde a la metodología del estudio es de corte cualitativo, ya que se aplicaron entrevistas semiestructuradas. Los objetivos del presente estudio consisten en apreciar el índice de criminalidad y violencia que existe en el Área Metropolitana de Monterrey, Nuevo León, identificar el nivel de participación ciudadana para prevenir delitos e indagar cuales son las causas o factores que influyen en las comunidades del área Metropolitana de Monterrey, para accionar de acuerdo a la Ley de Prevención Social de la Violencia y la Delincuencia con Participación Ciudadana del Estado de Nuevo León, así como comprobar la existencia de acciones sociales impulsadas por instituciones estatales que conlleven al fiel cumplimiento de la Ley de Prevención Social de la Violencia y la Delincuencia con Participación Ciudadana del Estado de Nuevo León, para efectos de una efectiva participación comunitaria para la prevención del delito.

Palabras clave: Participación ciudadana, prevención del delito, seguridad, seguridad ciudadana.

\section{ABSTRACT}

Crime prevention is the appropriate tool for reduction crimes in any society. This articles reviews citizen participation models and laws specially on security, mechanisms of prevention, with the objectives of determining the level of awareness of the population regarding the Law on Social Prevention of Violence and Crime with Citizen Participation of the State of Nuevo León and the level of citizen participation in crime prevention and to identify whether the social and government actions have been effective in crime prevention, as far as the methodology of the study is concerned, it is qualitative, since semi-structured interviews were applied. The objectives of this study are to assess the crime and violence index that exists in the Metropolitan Area of Monterrey, Nuevo León, identifying the level of citizen participation to prevent crimes and to investigate which are the causes or factors that influence the communities of the Metropolitan area of Monterrey, to act in accordance to the Law of Social Prevention of Violence and Crime with Citizen Participation in the state of Nuevo Leon, as well as verify the existence of social actions promoted by the state institutions that entail faithful compliance with the Law of Social Prevention and Crime with Citizen Participation of the state of Nuevo Leon, for the effects of an effective community participation in crime prevention.

Key words: Citizen participation, crime prevention, security, citizen security.

\footnotetext{
${ }^{1}$ El artículo es resultado de la tesis "Acciones comunitarias en la prevención del delito a raíz de "la ley de prevención social de la violencia y la delincuencia con participación ciudadana del Estado de Nuevo León" financiado por el Consejo Nacional de Científica y Tecnológica (CONACYT).

${ }^{2}$ Licenciado en Derecho por la Universidad Politécnica de Nicaragua (UPOLI). Candidato a Máster en Ciencias Políticas por la Universidad Autónoma de Nuevo León (UANL). Docente investigador. Becario del Consejo Nacional de Ciencia y Tecnología (CONACYT, México). Correo: davelopez217@gmail.com

${ }^{3}$ Doctor en Filosofía con Orientación en Ciencias Políticas por la Universidad Autónoma de Nuevo León (UANL). Profesor de tiempo Completo en la Facultad de Ciencias Políticas y Relaciones Internacionales de la UANL. Participa como investigador en el Laboratorio de Comunicación Política (LACOP). Es miembro del Sistema Nacional de Investigadores Nivel I. Correo: juan.martinezvll@uanl.edu.mx
} 


\section{INTRODUCCIÓN}

El incremento de la delincuencia y el miedo de la ciudadanía por ser victimario de un crimen son fenómenos que, al parecer, se dan en la mayoría de las sociedades contemporáneas, aun cuando las posibilidades, frecuencia y violencia que tienen sus habitantes de ser víctimas de un delito son distintas en los diferentes países (López Regonesi, 2000). En este sentido, se podría indicar que la inseguridad y violencia se presenta en cualquier país y su desarrollo depende del contexto específico de cada uno de ellos; en lo que corresponde a México estos fenómenos han incrementado de manera importante en las últimas décadas por lo que han pasado de la agenda pública a prioridad dentro de una agenda de gobierno.

En relación con lo anterior, la presente investigación trata de señalar la relevancia de la participación ciudadana en la prevención del delito tomando considerando al marco jurídico como referencia indispensable para la participación de los ciudadanos en la prevención de los delitos. A su vez, como preámbulo al tema de participación ciudadana es importante comentar que esta es naturalmente ligada a la democracia por ser la principal forma de gobierno que se sustenta en el diálogo e interacción entre los ciudadanos y los asuntos del Estado (Contreras \& Montecinos, 2019). Se habla de la participación ciudadana, para referir a la población integrante de una localidad determinada que coadyuva en coordinación con instancias administrativas en prosecución de actividades que satisfagan necesidades sociales, partiendo de una participación amplía hasta llegar a una participación más reducida como es la contribución comunitaria.

Retomando el tema de la inseguridad las acciones que se han tomado en México desde una perspectiva jurídica tienen su fundamento en su Constitución Política piedra angular de todo sistema jurídico de la cual se desprenden una variedad de leyes y regulaciones como sería el código penal federal, en el cual se presentan las políticas criminales para evitar y prevenir el delito, que se auxilia de otras leyes conexas, en lo que corresponde a la prevención del delito, existe la intervención activa e integradora de diferentes instancias como sería la participación ciudadana y no solo la intervención del Estado como principal sujeto garante. 
Por otra parte, en el artículo 2, primer párrafo de la Ley General del Sistema Nacional de Seguridad Pública (2017) indica que la finalidad del Estado, en cumplimiento de sus funciones, es salvaguardar la integridad y Derechos de las personas, y preservar las libertades, el orden y la paz pública, lo cual cumple con el sentido teleológico del Estado de Derecho. En lo que corresponde a la prevención del delito, las políticas públicas implementadas en cuestión de participación ciudadana presentan tres elementos que se consideran indispensables, como sería la mejoraría de la deteriorada relación entre comunidad y policía (acercamiento policial) que tendría como objetivo una intervención más activa de la ciudadanía en la prevención del delito, fortaleciendo el accionar policial.

Otro elemento sería el fortalecimiento las relaciones sociales existentes, bajo la premisa de que esto incrementaría el desarrollo y afianzamiento del capital social local, lo que contribuiría a prevenir la violencia. Finalmente, es indispensable que las políticas públicas de prevención del delito incluyan la participación e incidencia ciudadana en la prevención local del delito, tomando en consideración que los tipos y modalidades de la violencia son diferentes en cada localidad. La finalidad de este proceso de descentralización es otorga a los municipios un rol cada vez más activo en la formulación e implementación de dichas estrategias. (Dammert, 2002).

Según la historicidad que nos ofrece (Arellano \& Bernal, 2014) los últimos años que se han vivido en la sociedad mexicana ha sido afectada por varios factores criminológicos, lo que evidentemente ocasiona una serie de perturbaciones sociales como: la inseguridad en las calles, a propósito de esto, en México, la percepción de inseguridad ciudadana ha repercutido de forma directa, ya que las personas dejen de realizar actividades cotidianas, lo que genera la limitación del esparcimiento social, inhibe la posibilidad de generar cohesión social y, en algunos casos, genera otros problemas públicos de mayor envergadura (Jasso López, 2013).

Por otra parte, en lo que corresponde a Nuevo León la Fiscalía General de Justicia del Estado, reporta que los índices de criminalidad durante los últimos tres años (2016/2019) han incrementado de manera relevante, por lo que las autoridades competentes han impulsado la implementación de políticas públicas teniendo su sustento jurídico a través de la creación de leyes que tienen por finalidad la prevención del delito, no obstante, también se puede indicar 
que se ha generado un desgaste jurídico, puesto que, como lo menciona (Buendía, 2017) una de las principales causas por las que se incurre en un problema legal es el desconocimiento de la ley, es decir, la ciudadanía tiende a desconocer la implicancia e importancia de las mismas, es por ello que nace la necesidad de dar a conocer cuáles son estas normas y qué trascendencia tienen para efectos de constituir un resorte jurídico reciproco entre aplicación y ejecución por parte del Estado y la participación ciudadana en lo particular en la prevención del delito.

En lo que corresponde al marco jurídico que regula la seguridad pública y prevención del delito se tiene a bien destacar que el origen de todo sistema jurídico es su Constitución Política, por lo que es relevante indicar que ninguna otra ley, norma o disposición legal puede, en ningún caso, contradecir a la Constitución Política, lo que en Derecho se conoce como el control Constitucional de la norma partiendo del principio de Supremacía Constitucional (López Mejía, 2018), es en este sentido, México en materia de seguridad pública y prevención del delito contempla pautas legales situadas en artículo 21, párrafo noveno de su Constitución Política que textualmente expresa lo siguiente:

La seguridad pública es una función del Estado a cargo de la Federación, las entidades federativas y los Municipios, cuyos fines son salvaguardar la vida, las libertades, la integridad y el patrimonio de las personas, así como contribuir a la generación y preservación del orden público y la paz social, de conformidad con lo previsto en esta Constitución y las leyes en la materia. La seguridad pública comprende la prevención, investigación y persecución de los delitos, así como la sanción de las infracciones administrativas, en los términos de la ley, en las respectivas competencias que esta Constitución señala. La actuación de las instituciones de seguridad pública se regirá por los principios de legalidad, objetividad, eficiencia, profesionalismo, honradez y respeto a los Derechos humanos reconocidos en esta Constitución. (Constitución de los Estados Unidos Mexicano, 2020, p. 25).

Como se puede apreciar en la Carta Magna se presenta el concepto de seguridad pública, el cual comprende la prevención, investigación y persecución de los delitos, creando así el Estado un Derecho de total exigibilidad por parte de los ciudadanos, no obstante, no es 
suficiente la manifestación escrita en la Constitución Política del país referente a este tema, es por eso que la Cámara de Diputados del Honorable Congreso de la Unión, en el 2009 aprobó la Ley General del Sistema Nacional de Seguridad Pública, con la finalidad de exponer de manera específica el contenido y significado de la seguridad publica en México, misma que a continuación se detalla:

\section{Ley General del Sistema Nacional de Seguridad Pública}

Esta normatividad jurídica de rango federal abarca de una forma amplia el significado de la seguridad pública y lo que conlleva, pues, en su artículo segundo contiene un parafraseo similar de lo que dispone el artículo 21 parágrafo noveno de la Constitución Política de los Estados Unidos Mexicanos; no obstante, en el desarrollo del artículo se manifiesta textualmente lo siguiente:

El Estado desarrollará políticas en materia de prevención social del delito con carácter integral, sobre las causas que generan la comisión de delitos y conductas antisociales, así como programas y acciones para fomentar en la sociedad valores culturales y cívicos, que induzcan el respeto a la legalidad y a la protección de las víctimas (Ley General del Sistema Nacional de Seguridad Pública, 2019, p.1)

De igual forma, el artículo séptimo dispone que las Instituciones de Seguridad Pública de la Federación, las Entidades Federativas y los Municipios, deberán coordinarse para determinar la participación de la comunidad y de instituciones académicas en colaboración con los procesos de evaluación de políticas de prevención del delito, así como de las Instituciones de Seguridad Pública, a través de mecanismos eficaces.

Ahora bien, de una forma más delimitante, pero siempre con relación a la Constitución Federal, se encuentra la Constitución Política del Estado de Nuevo León y que en su artículo 25 párrafo sexto dispone textualmente lo siguiente: "El Estado, en ejercicio de la función de seguridad pública, deberá en todo momento salvaguardar la integridad y Derechos de las personas, e igualmente preservará las libertades, el orden y la paz públicos”. De esta se desprende la Ley de Prevención Social de la Violencia y la Delincuencia con Participación Ciudadana del Estado de Nuevo León que tiene como objetivo desarrollar en el Estado, las 
bases para la coordinación en materia de prevención social de la violencia y de la delincuencia con participación ciudadana acorde con los Sistemas Nacional, Estatal y Municipal de Seguridad Pública, que se prevén en los artículos 21 de la Constitución Política de los Estados Unidos Mexicanos; 25 de la Constitución Política del Estado Libre y Soberano de Nuevo León, 2 y demás relativos de la Ley General del Sistema Nacional de Seguridad Pública; 1 y demás relativos de la Ley General para la Prevención Social de la Violencia y la Delincuencia, y en todo lo relativo a la Ley de Seguridad Pública para el Estado de Nuevo León.

Este cuerpo jurídico es el sustento para una serie de modelos y mecanismos tendientes a la participación ciudadana y comunitaria en pro de la prevención del delito, así como las instancias de coordinación del sistema estatal de prevención, programas, planes y acciones que se promuevan para disminuir la criminalidad y la violencia mediante la participación ciudadana y comunitaria y que estos mismos a través de los planes y programas llevado a cabo por el municipio puedan identificar los factores generadores de la violencia y la delincuencia dentro de las comunidades.

Los objetivos planteados en la siguiente investigación son determinar el grado de conocimiento de la población con respecto a la Ley de Prevención Social de la Violencia y la Delincuencia con Participación Ciudadana del Estado de Nuevo León y los niveles de participación ciudadana en la prevención del delito. Asimismo, se busca identificar si las acciones sociales y gubernamentales han sido eficientes en la prevención del delito; además se trata de identificar las causas o factores que influyen en las comunidades para accionar a favor de la prevención del delito.

Por otra parte, el presente artículo está dividido en cuatro apartados, el primer apartado corresponde al desarrollo del marco teórico donde se revisa la literatura relacionada con la prevención del delito, seguridad y participación ciudadana; en un segundo momento la metodología del estudio, en tercera instancia los análisis de resultados para finalmente pasar a la sección de discusión y conclusiones. 


\section{DESARROLLO DEL TRABAJO}

\section{Participación ciudadana}

Desde una perspectiva científica, se define la participación como un componente elemental de la democracia, como una forma de organización social y de gobierno, además refiere que en las ciudades existen diferentes formas de participación: social, comunitaria, ciudadana, política y que todas son necesarias para hacer de los individuos que la habitan ciudadanos con Derechos y obligaciones sociales, políticos, urbanos (Ziccardi, 2004).

Ahora bien, desde un aspecto más pragmático, postula Sanhueza que "la participación de la ciudadanía ayuda a la redefinición de lo público, quitándole el uso exclusivo de lo público al Estado y propiciando conquistas al margen del mercado y el Estado” (2004, pág. 2), de modo que, la sociedad en su ámbito colaborativo con el Estado genera un vínculo armonioso que coadyuva en modificar de forma positiva en la estratificación social y permiten eliminar las carencias de índoles públicas. Si bien es cierto que la seguridad pública es una función a cargo del Estado, esta también implica un compromiso social el cual debe ser asumido por toda la sociedad.

En este sentido, la participación ciudadana, guarda relación con el desarrollo humano, no podríamos entender un acto que denote tolerancia, solidaridad, humanismo, subsidiariedad y demás valores de trascendencia fundamental en el individuo, que no tenga como fin primordial, el desenvolvimiento holístico del ser humano dentro de una sociedad. Por lo anterior, se podría indicar que la participación ciudadana es la constante búsqueda del individuo dentro de la sociedad para lograr una mejor calidad de vida, es la respuesta a promesas incumplidas y a su vez es la alerta para despertar una sociedad dormida.

Por otra parte, la participación comunitaria está orientada al conjunto de acciones desarrolladas por diversos sectores comunitarios (barrios, colonias, vecindarios, etc.), en la búsqueda de soluciones a sus necesidades específicas. Asimismo, la comunidad implica un cierto tipo de realidad social en la que están presentes algunos actores vitales, donde se pueden destacar los siguientes: 
1. Existencia de un colectivo humano al que se le reconoce capacidad de Derechos y obligaciones, así como protagonista de acciones y decisiones, con voluntad de incidir en el cambio y mejora de condiciones de vida de las personas que forman parte de él.

2. Existencia de sentimiento de pertenencia, es decir, de un cierto grado de integración subjetiva en una identidad comunitaria compartida.

3. Existencia y arraigo a un territorio, a un cierto espacio compartido que articula a agentes, instrumentos y contenidos para la acción. Un espacio físico, una geografía, que incorpora significados de pertenencia.

A manera de resumen, la comunidad adquiere sentido cuando se desarrolla a partir de un colectivo humano que comparte un espacio y una conciencia de pertenencia, que genera procesos de vinculación y apoyo mutuo, y que activa voluntades de protagonismo en la mejora de su propia realidad (Gomá, 2008).

El espacio local se encuentra en constante progreso, pues durante mucho tiempo ha empleado una lucha constante en búsqueda de la descentralización y de romper el esquema de ejecutor del Estado Benefactor. El espacio local para poder dar la respuesta a los ciudadanos participativos requiere de nuevos enfoques, en donde la toma de decisiones pueda ser inmediata y no controlada desde otros niveles de acción (Guillen, 2009). En lo que corresponde a la prevención del delito, es importante a destacar que muchas de las conductas delictivas tradicionales que se desarrollan en nuestra comunidad, no dependen tanto de la habilidad del delincuente para realizarlas, quien solo funge como un ente oportunista; sino que se deben a la falta de previsión y pericia tanto de quienes se encargarían de brindar la seguridad al ciudadano como del mismo ciudadano, quien no auxilia a los órganos encargados al no prevenir ni denunciar el delito y que además le recrimina al Estado, que, sin darse cuenta él es quien se ha vuelto un facilitador de su propia victimización.

La necesidad de abordar este tema de prevención del delito, resulta de alta importancia, en virtud que, por el simple contexto literario de la palabra prevención se pueden extraer aspectos positivos por su significado, para esto la (Real Academia Española, 2019) nos dice que la prevención es "preparación y disposición que se hace anticipadamente para evitar un riesgo o ejecutar algo" esto nos indican como conocimiento a priori los elementos distintivos 
que contiene tal expresión, por lo que al momento de realizar la conjugación gramatical con otras palabras, esto conlleva a la personalización de la prevención, en el caso que nos ocupa, lo asociaremos con temas relacionados con el delito y todas sus vinculaciones pertinentes y, tomando en consideración lo ya dicho por (Sozzo, 2000) nos vincula la prevención con el delito y dice que: "Prevenir el delito es la intervención ex - ante, antes que el delito se produzca" y partiendo de esta expresión es de total trascendencia ir delimitando conceptual y doctrinariamente estas palabras, a saber:

¿Qué es el delito?

Concepción Etimológica del Delito: el termino delito etimológicamente proviene del verbo latino delinquo, delínquere, deliqui, delictum. Se formó el sustantivo delictum, i, que tenía el significado de los pecados de omisión. Por qué lo hemos de traducir como falta, error, descuido (Arnal, 2017).

Concepción Dogmática del Delito: surge científicamente en las Teoría de las Normas de Binding, que preceptúa que el delincuente vulnera el supuesto hipotético de la norma jurídica, no la ley. La organización que se considera más eficaz para el estudio dogmático de los estudios penales deriva de la teoría pentatónica de cincos elementos; según esta teoría podemos definir al delito como un hecho, típico, antijurídico, culpable y punible (Calderón, 2015). Dicho de otra forma, para que concurra el delito debe de existir primero una conducta o un hecho realizado por un individuo, la misma puede constar de una acción u omisión que previamente este tipificada por la ley como una prohibición de hacer (acción) o una obligación de hacer (omisión).

Concepción Sociológica del Delito: Según Rafaél Garófalo discípulo de Cesar Lombroso (padre de la criminología), en su obra Criminología (Turín, 1887) manifestaba que el delito ha sido concebido como una lesión de los sentimientos altruistas y fundamentales de piedad y probidad en la medida media en que son poseídos por la comunidad y en la medida media en que son indispensables para la adaptación del individuo a la sociedad. Por otro lado, y en estudios más recientes, Luis Garrido (1996) manifiesta que a la sociología criminal es aquella a la que le incumbe poner en claro la gestación y desarrollo del delito, relacionándolo con los 
factores y productos colectivos en cuanto lo establecen, y también le toca indicar los efectos que a su vez produzca la infracción, tanto en la estructura como en la dinámica social.

Por otra parte, es importante comentar que la prevención puede ser como conicidad según (Dijk, 1990) como: políticas, medidas y técnicas, utilizadas fuera de los límites de sistema de justicia penal, y dirigidas a la reducción del daño producido por aquellas acciones definidas como delitos por el Estado. La prevención de delito es una herramienta transversal para disminuir los indicies de criminalidad, pero para ello debemos de conocer científicamente el contexto de la palabra prevención y (Marisol, 2003) nos refiere que etimológicamente el vocablo prevención, "proveniente del latín preventione indica la acción o efecto de prevenir, disponer, organizar y prever para evitar un riesgo" (p.165). De tal manera, se entiende a la acción de prevenir el delito como aquellas acciones y estrategias encaminadas a reducir el riesgo de que se produzcan delitos y a su vez disminuir que los efectos perjudiciales para la ciudadanía y la sociedad sean factores que limiten el temor a la delincuencia, y en consecuencia se puedan prevenir e influir en las múltiples causas delictuosas.

En este mismo sentido, la Organización de las Naciones Unidad en su resolución 2002/13 nos define la «prevención del delito» como aquella prevención del crimen que engloba las estrategias y medidas que buscan reducir los riesgos de la infracción y los efectos perjudiciales que estos últimos pudiesen causar en las personas y en la sociedad, incluyendo la sensación del miedo al crimen, combatiendo para ello sus múltiples causas (2002). De tal suerte que la forma preventiva del crimen necesita según Waller (1997) reunir a los responsables de la vivienda, de los servicios sociales, del tiempo libre, de las escuelas, de la policía y de la justicia para poder enfrentar situaciones que conducen a la delincuencia (p.76).

Asimismo, en lo que corresponde a la prevención del delito se pueden presentar diferentes modelos; entre los cuales se encuentra el modelo punitivo que para su entendimiento es necesario indagar en el término punibilidad que como lo expresa el penalista filosofo Rodríguez Collado la punibilidad, suele ser entendida, como posibilidad abstracta de imponer un castigo, ya como posibilidad concreta de aplicarlo, lo que se podría traducir que en el primer sentido alude al delito y a la pena en su momento normativo, esto es como instituciones estrictamente jurídicas, en el segundo hace referencia al hecho delictivo y a la 
sanción en un plano fáctico, es decir, como fenómenos que se materializan en el mundo real (1995, p.260). Cabe mencionar que el modelo punitivo es uno de los sistemas más antiguo que el Estado ha tomado como medida castigadora mediante el uso de la fuerza, Hobbes afirmaba que el sujeto que atenta contra la seguridad o la supervivencia del Estado no es un criminal sino un adverso del Estado y para él no valen las reglas del Derecho sino los de la coacción o la fuerza (1651). Podemos decir que dentro de los modelos punitivos existen mecanismos jurídicos que el Estado como agente garantista tiene la obligación de imponer medidas coercitivas a los infractores de la ley, es así que, por medio de este elemento punitivo, busca la persecución de la paz y el bien común de la sociedad, donde la inclusión del miedo a ser castigado sea fundamental para la disminución delictiva. En otras palabras, la sanción o castigo demuestra el poder punitivo del orden jurídico e institucional sobre cualquier sujeto de la sociedad que pretenda perturbar el discurso y los quehaceres estatales y la tranquilidad de las víctimas.

Dicho lo anterior, es preciso señalar que "en el modelo del control punitivo, el Estado tiende a desarrollarse mediante normas vinculantes que funcionan como un contrato social que prioriza el respeto a derechos y libertades de cada ciudadano a través del cumplimiento de normas y leyes que miden el compromiso de cada individuo con la sociedad, y su respeto a la misma" (Vega, 2017, pág. 153). Autores como Rodríguez \& Montero (2012) defienden este modelo y dicen que la incrementación de las medidas y penas privativas de libertad tendrán consecuencias repulsivas para los infractores de la ley. Por otro lado, existen enfoques actuales que defienden este modelo punitivo agregando que la mejor forma para prevenir el delito es por medio de la vigilancia continua de patrullas policiales, la observación de personas y cosas, así como su intervención en cualquier incidente.

Por otra parte, en el modelo social se trata de construir programas o políticas públicas de desarrollo general e integral, mismas que pueden incorporarse en iniciativas con características multisectoriales al servicio e intereses de la comunidad, por ejemplo, la creación de nuevos espacios productivos mediante empleos, educación, protección de la juventud, urbanismo, vivienda, salud, inclusión social, sistemas policiales y de justicia (Chalom, Léonard, Vanderschueren, \& Vézina, 2001). 
La prevención social del crimen o delito, busca cambiar las motivaciones criminales que son observadas como algo que habita en las personas más que en las cosas, dentro de un ambiente social; por lo que podemos decir que la prevención social "busca alcanzar esto a través de medidas típicas de la política social como políticas de vivienda, educación y tratamiento sanitario con respecto al alcohol y el delito; política familiar y educativa, trabajo para los jóvenes y políticas de empleo" (Gilling, 1998, pág. 64). Como lo expresa Cusson citado por Rivera Ciriaco (2018) "la prevención social tiene sus lineamientos en estrategias dirigidas a las causas de los delitos con la finalidad de disminuir su incremento o aparición, por lo que pretende más evitar el delito a través del desarrollo social o humano" (pag.88), A su vez autores como De Cauter \& Walgrave (1999) refieren que la prevención social, no es una acción determinada o una de las numerosas formas preventivas, sino una política global que busca el bienestar social de todos los sectores de las políticas administrativas. Lo que se podría decir que el factor integrante clave de este modelo es el Estado por que tiene un roll más participativo y ofertante de políticas gubernamentales que integren a la sociedad a combatir el delito mediante la aparición de programas y proyectos tendientes a eliminar situaciones peligrosas que conlleven al crimen.

Este modelo requiere de una inversión económica gubernamental, puesto que consiste en el involucramiento de la sociedad en programas estatales, federales o municipales en la acción de reducir el delito y para la ejecución concreta del mismo se requiere de movilidad presupuestaria ya que este modelo se lleva a cabo por políticas públicas que involucren causas sociales-económicas, motivacionales, comunitarias y situacionales que influyen en la prevención de delitos. Este modelo defiende que los defectos del sistema coercitivo son los que potencian el crimen y que para corregir o disminuirlo es primeramente importante sanar aquellas carencias de los modelos sociales para que influyan de forma benéfica sobre los actos individuales o colectivos.

En relación con el modelo comunitario, se puede indicar que se ejecuta mediante acciones en pro de mantener la participación de los ciudadanos en materia de prevención, indistintamente sea a título individual o colectivo, por ejemplo, el respaldo social a la comunidad, mejora del ambiente físico y social, hasta llegar a la reducción de oportunidades del delincuente a través de formas diversas de vigilancia llevadas adelante por parte de los mismos ciudadanos 
(Selmin, 2009). Se habla también de modelo comunitario como el que difunde una propaganda de cooperación entre las fuerzas de seguridad gubernamentales y la población, en función de concientizar a la colectividad de la importancia y necesidad que juega su papel en el auxilio eficaz con la policía. A través de este método muchos delitos podrían ser evitados si la ciudadanía fuera más consciente de las secuelas que generan las actividades criminales en su entorno físico.

Se ha fundamentado según diversas literaturas científicas que el crimen puede disminuirse si se incrementan los riesgos potenciales de que el criminal pueda ser captado in fraganti a través de más infraestructuras y vigilancia que la que ya proporcionan las fuerzas de seguridad del Estado y todo esto se puede lograr a como refiere Garland (2001), a través de la cooperación social desarrollada por una mejor comunidad organizada, la introducción de patrullas ciudadanas en el barrio; esto potencia una mejor relación entre residentes, así como entre la policía y la población de tal suerte que, a la medida que se implemente medidas de seguridad en las comunidades y cooperación social, se limitarían de forma incrementada las posibilidades en que un sujeto con intenciones antisociales pueda delinquir.

Finalmente el modelo situacional tiene como finalidad reducir oportunidades delictivas y disminuir la percepción de miedo al crimen e inseguridad de las comunidades en espacios determinados, mediante estrategias dirigidas a modificar algunos factores y condiciones de riesgo físico y ambiental, por medio de políticas (acciones) dirigidas a la protección del blanco y al diseño del entorno urbano, regenerando y creando espacios públicos de calidad, seguros, integradores, que acojan la diversidad de funciones y usuarios (Ministerio de Vivienda y Urbanismo en colaboración con el Ministerio del Interior y Seguridad Pública, Chile, 2013).

Este sistema señala prevenir los delitos reduciendo las oportunidades de ejecutarlos, incrementando para los delincuentes el riesgo de ser encontrado cometiendo un delito y reduciendo al mínimo los beneficios potenciales. Para el Centro internacional para la prevención de la criminalidad (2010) la prevención situacional busca limitar las posibilidades de cometer delitos "incrementando el esfuerzo" que debe llevar a cabo el criminal, “incrementando el riesgo" de exposición en la comisión de violación a la ley, principalmente 
por medio del ordenamiento y visibilidad del entorno. El triunfo de esta estrategia depende de la posibilidad de que los delincuentes sean efectivamente afectados por las intervenciones sobre la situación y el espacio, de manera que perciban a estos elementos como influencias adversas con respecto a la facilidad, el riesgo o las recompensas de la realización de los delitos (Sozzo, 2000).

\section{Preguntas de investigación}

PI1.: ¿Cuál es el grado de conocimiento de la ciudadanía respecto a la Ley de Prevención Social de la Violencia y la Delincuencia con Participación Ciudadana del Estado de Nuevo León?

PI2.: ¿Cuál es el nivel de participación ciudadana en la prevención del delito?

PI3.: ¿Qué tan eficientes han sido las acciones sociales y gubernamentales en la prevención del delito?

PI4.: ¿Cuáles son los factores que influyen en las comunidades para que éstas actúen a favor o en contra de la prevención del delito?

\section{METODO}

Para la presente investigación de corte cualitativo se llevaron a cabo entrevistas semiestructurada a tres expertos en materia jurídica y política, los entrevistados a su vez se han desarrollado dentro de líneas de investigación de participación ciudadana y prevención del delito.

\section{Instrumento}

El instrumento aplicado fueron entrevistas semiestructuradas que nos permitieron extraer datos e información de expertos, atendiendo que "la entrevista por su naturaleza busca entender al mundo desde la perspectiva del entrevistado, y desmenuzar los significados de sus experiencias" (Álvarez-Gayou, 2003, p.109). Asimismo, al ser una entrevista semiestructurada nos permite de acuerdo con el avance de esta y las respuestas espontaneas 
del entrevistado modificar la secuencia, la forma y el contenido de la pregunta dándonos la oportunidad de obtener de manera más amplia los conocimientos o postura que tiene el consultado sobre el tema en lo particular.

\section{Análisis de resultados}

En relación con las respuestas emitidas en las diferentes entrevistas realizadas se hicieron las siguientes categorías: ley, ciudadanía, acción de gobierno en prevención del delito y participación. El programa utilizado para el análisis de datos fue NVIVO 13. Como se muestra en figura No. 1.

Figura No. 1

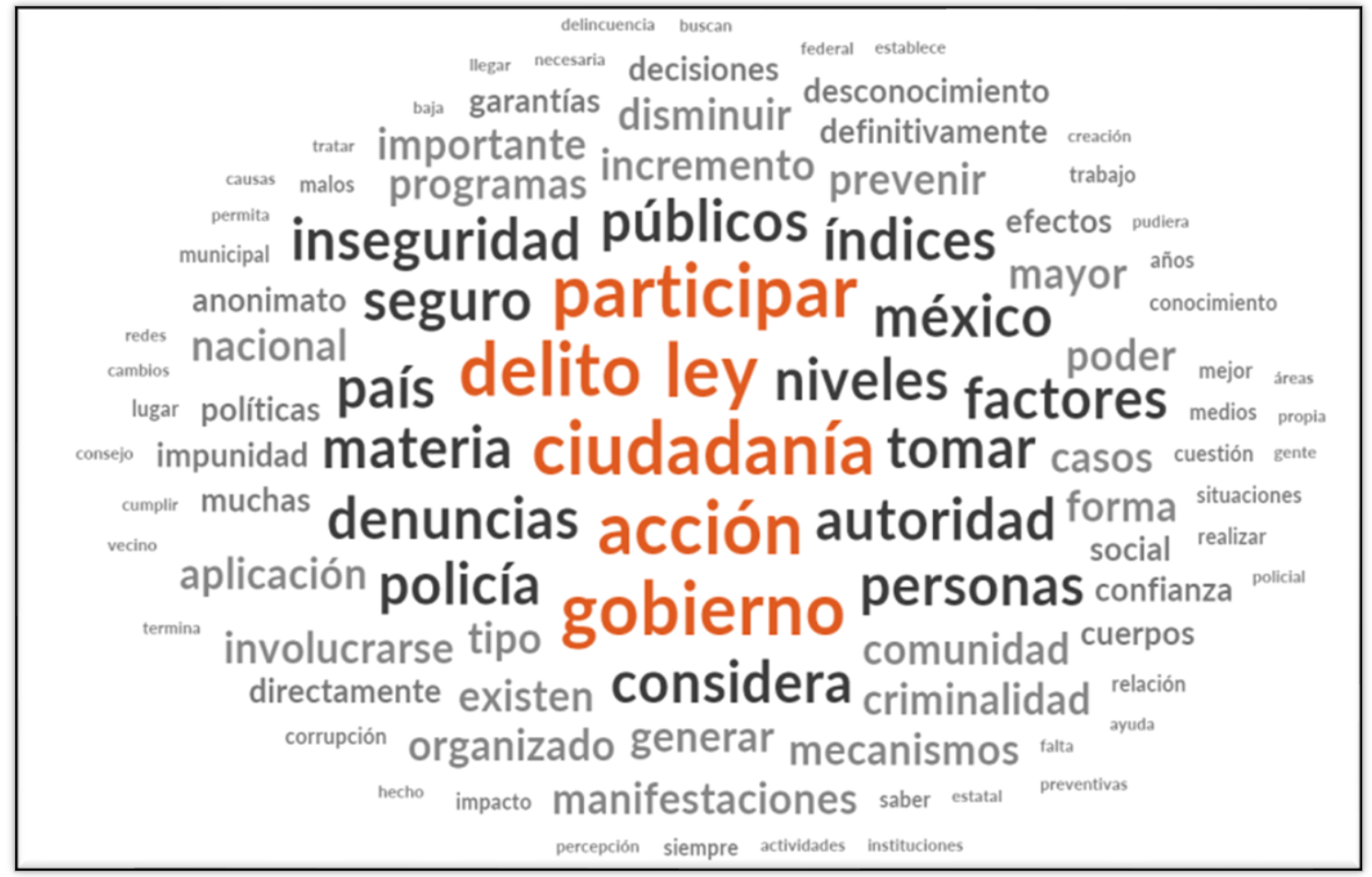

Fuente: elaboración propia con datos extraídos de NVivo13

Con relación al concepto de ley, los entrevistados nos indican que existe un desconocimiento de la ley por parte de los ciudadanos a causa de que el gobierno no le da la promoción debida en comparación con otros programas. Asimismo, desde su perspectiva consideran que la ley se encuentra bien estructurada y cumple con el deber ser en relación con la prevención del 
delito, pero lamentablemente el desconocimiento de la misma no permite que se puedan llevar a cabo los objetivos para lo cual fue creada.

En cuanto a las expresiones explicitas de los expertos quedan sustento a lo anterior se encuentran las siguientes:

Entrevistado 1: ....se debe de aplicar la ley, pero también debe la ciudadanía tener el conocimiento de la ley, hay un número alto de casos que desconocemos la ley... ...la autoridad debe de hacer alguna campaña que invite a los ciudadanos a conocer la ley...

Entrevistado 2: ... de nada sirve una ley que pudiera estar creada si no es eficiente... ...podemos tener una ley perfectamente estructurada, clara con todos los mecanismos, pero de ahí a que se lleve a la práctica, es ahí donde esta el conflicto...

Entrevistado 3: ... pero si no la conocemos, por más que la ley este bien trabajada, sin lagunas o detalles... ... se debe una buena publicidad para que llegue a todos... ...no es nada más de la ciudadanía sino también de los funcionarios...

En el análisis del término ciudadanía los expertos indicaron que el gobierno debe de generar confianza para que el ciudadano pueda participar, asimismo esta confianza es necesaria para que se creen lazos entre la sociedad civil y el gobierno y de esta manera poder incidir en la prevención del delito. De acuerdo con los entrevistados dentro de las garantías que deben estar incluidas en una ley de prevención del delito es el anonimato en las presentaciones de denuncias; además consideran que existe por parte del ciudadano una desafección y descontento con relación a la aplicación de la ley por parte de la autoridad competente.

Entrevistado 1.: ... debe ser una denuncia que quede en el anonimato... ... debe de generar confianza en los ciudadanos para que estos se sientan seguros al actuar con relación a la prevención del delito... ... hay una desconfianza y pues esto dificulta el involucramiento del ciudadano...

Entrevistado 2.: ... hablamos de un desconocimiento, de una desafección política... ...no hay interés... ...no son escuchados por parte de los gobernantes... ...un 
descontento social, por parte de la ciudadanía con relación a las acciones gubernamentales, independientemente del nivel...

Entrevistado 3.: ...que exista garantías para los ciudadanos que participen en el procedimiento de denuncia... ...apoyarse entre vecinos hacia la denuncia y ser valiente si vemos una conducta que no es correcta... ...no que hacemos caso omiso...

En correlación con acción de gobierno los expertos manifestaron que las acciones de gobierno como campañas, programas, consejos de seguridad, integración de agrupación y comités vecinales, inclusión social, educación, deporte, la promoción de la ley existente son estrategias preventivas del delito que deberían de ejecutar las autoridades. Por otra parte, la corrupción, impunidad y tráfico de influencia son acciones que impiden la participación ciudadana para la prevención del delito.

Entrevistado 1.: ...generar más espacios para los jóvenes, incrementar las oportunidades de nivel educativo, las oportunidades de empleo, mejorar el nivel de vida, involucrar a los ciudadanos y hacerlos conscientes de lo que está sucediendo en el país...

Entrevistado 2.: ...consejo de seguridad, hay participación de los presidentes municipales en conjunto con el gobierno estatal, que toman o son participes en la toma de decisiones... ...en este consejo de seguridad, participan representantes ciudadanos en estas acciones o en estas reuniones ... ...que toman diversas decisiones para atacar esta inseguridad... ...con una mayor participación con los ciudadanos en prevención, en pláticas, actividades deportivas para jóvenes, ciudadanos... ... publicitar en los medios para tratar de incluir más a la ciudadanía con programas de prevención del delito...

Entrevistado 3.: ...la mercadotécnica siempre funciona, o sea que haya un buen spot, un buen nombre ese programa para que la gente se puede identificar y acceder a él... 
Por otra parte, en lo que corresponde a la categoría de participación, se obtuvieron los siguientes desenlaces: hay una falta de participación activa de la ciudadanía para la prevención del delito; además corroboran que efectivamente existe una ley de participación en el Estado que es desconocida por la mayoría de la ciudadanía, también reportan que la participación crea cadenas y lazos entre los ciudadanos que ayudan en la prevención del delito.

Entrevistado 1.: ...Si no hay confianza en las autoridades, dificilmente va a ver denuncias y por ende, no va a ver participación de la ciudadanía... ...si no hay confianza pues no hay involucramiento y obviamente resultados... ... si existe la ley de prevención del delito...

Entrevistado 2.: ....si efectivamente existe una ley de participación ciudadana en la entidad, específicamente en Nuevo León, que esta señala mecanismos, que los ciudadanos pudiésemos utilizar... ...tanto en materia de seguridad, como en otros temas...

Entrevistado 3.: ...es apática la ciudadanía. No participa...

\section{Discusión}

Los resultados de la investigación permiten el logro de los objetivos marcados para el estudio, ya que se pudieron identificar el grado de conocimiento que tiene la población con respecto a la Ley de Prevención Social de la Violencia y la Delincuencia con Participación Ciudadana del Estado de Nuevo León, los niveles de participación ciudadana en la prevención del delito, acciones sociales y gubernamentales que podrían ser eficientes en la prevención del delito y las causas o factores que influyen en las comunidades para accionar a favor o no de la prevención del delito.

De igual forma nos permitió dar respuestas a las preguntas de investigación planteadas en el presente estudio. 
PI1.: ¿Cuál es el grado de conocimiento de la ciudadanía respecto a la Ley de Prevención Social de la Violencia y la Delincuencia con Participación Ciudadana del Estado de Nuevo León?

En relación con los resultados obtenidos se puede indicar que existe una ley de prevención del delito en la entidad pero es desconocida por la mayoría de los ciudadanos, en este sentido se puede afirmar que este desconocimiento no permite el logro de los objetivos de la misma que son desarrollar las bases para la coordinación en materia de prevención social de la violencia y de la delincuencia con participación ciudadana acorde con los Sistemas Nacional, Estatal y Municipal de Seguridad Pública, que se prevén en los artículos 21 de la Constitución Política de los Estados Unidos Mexicanos; 25 de la Constitución Política del Estado Libre y Soberano de Nuevo León, 2 y demás relativos de la Ley General del Sistema Nacional de Seguridad Pública; 1 y demás relativos de la Ley General para la Prevención Social de la Violencia y la Delincuencia. Dentro de las causas del desconocimiento de la ley se pueden considerar la ausencia de promoción por parte del gobierno; es claro que está publicado en el periódico oficial del Estado de Nuevo León pero esta acción no es suficiente para que la mayoría de la ciudadanía se entere de su existencia, por lo que se considera necesario su promoción por medios de comunicación masivos, pues una de las principales causas por las que se incurre en un problema legal es el desconocimiento de la ley (Buendía, 2017).

PI2.: ¿Cuál es el nivel de participación ciudadana en la prevención del delito?

De acuerdo los resultados analizados se pueden reportar que la participación de la ciudadanía en la prevención del delito es baja, en este sentido es importante recalcar la importancia que tendrían la participación ciudadana en la generación de redes que permitan la prevención del delito, ya que "prevenir el delito es la intervención ex - ante, antes que el delito se produzca" (Sozzo, 2000. Pág. 104) Se podría decir que la prevención en sentido amplio consiste en preparar o disponer lo necesario anticipadamente, para evitar un riesgo o la materialización de un determinado evento en el futuro; en nuestro caso, la prevención serían las sumas de políticas tendientes a impedir el surgimiento o avance de la criminalidad (Galindez Delvitto, 2013) de ahí la relevancia de la intervención de la ciudadanía en este tipo de actividades; si 
bien es cierto la seguridad pública es una función a cargo del Estado, ésta también implica un compromiso social el cual debe ser asumido por toda la sociedad.

PI3.: ¿Qué tan eficientes han sido las acciones sociales y gubernamentales en la prevención del delito?

En el análisis de los datos extraídos en las entrevistas de los expertos se pudo satisfacer con esta pregunta en el siguiente sentido: las acciones sociales y de gobierno en pro de la prevención del delito son ineficientes debido que no hay campañas, programas, consejos de seguridad, integración de agrupación y comités vecinales, inclusión social, educación, deporte, promoción de las leyes efectivas como algunas estrategias preventivas del delito y por el contrario, la existencia de corrupción, impunidad y tráfico de influencia, entre otras, son acciones que impiden una participación activa ciudadana para prevenir actividades delictuosas, en el entendido que la prevención del delito se puede definir como las políticas, medidas y técnicas que se utilizan con el fin de prevenir las acciones definidas como delitos por el Estado (Dijk, 1990). De esta situación se desprende un claro desafío para las políticas públicas de prevención, las que requieren de un contacto permanente con la población y de campañas de información sobre las acciones realizadas (Dammert, 2002). La literatura asociada y en particular el Centro Internacional para la Prevención de la Criminalidad dispone que las estrategias de prevención del delito son las formas más viables de reducir la delincuencia y los delitos violentos con presupuestos reducidos. Una manera efectiva de evitar "reinventar acciones" en la prevención del delito es usar estrategias desarrolladas exitosamente por los gobiernos y ciudades donde han sido aplicadas en Europa, Latinoamérica, Australia, Asia y Estados Unidos (1995).

PI4.: ¿Cuáles son los factores que influyen en las comunidades para que éstas actúen a favor o en contra de la prevención del delito?

En cuanto esta última pregunta se comprobó mediante el estudio de resultados que el gobierno debe de generar confianza para que el ciudadano pueda participar, debido que es por medio de la confianza que los ciudadanos podrían crear lazos entre la sociedad civil y el gobierno, en este sentido, se puede decir que esta sería una de las principales causas que influye en la ciudadanía para prevenir el delito, pues, según lo analizado la población se ve 
muchas veces impedida de participar por miedo y desconfianza de las instituciones policiales y gubernamentales; en cuanto a la prevención también hay una limitación de denunciar algún delito o bien prevenirlo por la causa principal de quedar expuesto ante el posible agresor, pues es preciso indicar que en materia de seguridad pública, los estudios psicológicos de las emociones han identificado la ansiedad, el enojo, la preocupación, el miedo y la desconfianza como factores de estrés referentes a experiencias de agresión, robo, secuestro, extorsión, asesinato o violación (Fernández \& Revilla, 2010) por lo que los expertos recomiendan que el anonimato en las presentaciones de denuncias y garantías de seguridad, sería una forma efectiva de que los ciudadanos se involucren en pro de la prevención del delito, de igual forma se comprobó que otra causa que influye en la ausencia de participación ciudadana en la prevención del delito es la desafección y descontento en relación con la aplicación de la ley por parte de la autoridad competente, en este sentido Montero vincula el descontento con el desencanto y lo define como la molestia resultante de la creencia que la actuación del gobierno es insuficiente respecto a los deseos o expectativas de los ciudadanos lo que en materia de prevención es negativo pues limita la participación ciudadana (1998 p. 26).

\section{CONCLUSIONES}

Finalmente, podemos mencionar que la participación ciudadana en la prevención del delito es precaria, esto debido a factores internos del ciudadano que son provocados por factores externos de su entorno social y gubernamental, pues, no hay una promoción efectiva y eficaz de la ley de prevención social de la violencia y la delincuencia con participación ciudadana del Estado de Nuevo León por parte del Estado, tanto el ciudadano como la autoridad competente desconoce el contenido de la ley y este desconocimiento provoca conflictos sociales, todo lo contrario al espíritu mismo de la ley, que es la prevención del delito mediante la participación activa de la ciudadanía. Partiendo de este problema la solución que se sugiere producto de la presente investigación es que el gobierno promueva campañas de promoción en medios de comunicación masiva tendientes a dar conocimiento de forma flexible y amigable a los ciudadanos con respecto a las acciones que pueden tener en pro de prevenir acciones delictuosas. Por otro lado, podemos mencionar que la ciudadanía tiene responsabilidad compartida con el Estado de crear lazos con la finalidad de intervenir, notificar y accionar para la prevención del delito y por otro lado el Estado debe de asegurar 
a los ciudadanos ciertas garantías que permitan generar confianza y seguridad al momento de actuar conforme la mentada ley, también debe de generar acciones gubernamentales con vinculación social, que atienda temas de entornos sociales y demandas ciudadanas para que de esta manera se puedan recuperar espacios públicos y capital humano que se encuentra descarriado y actuando ilegítimamente en la sociedad.

\section{REFERENCIAS}

Álarez-Gayou Jurgenson, J. (2003). Cómo hacer investigación cualitativa. Fundamentos y Metodología. México: Paidós.

Álvarez Enrique, L. (2014). La política de Derechos del Gobierno del Distrito Federal. Acta Sociológica, 35-69.

Arellano, E., \& Bernal, R. (2014). Evaluación de la Seguridad Pública. Centro de Estudios Sociales y de Opinión.

Arnal, M. (2017). Una palabra cada día. El almanaque y la casa del libro, 2.

Buendía, Á. (2017). Desconocimiento de las leyes, principal causa de conflictos legales. Notimex.

Calderón, A. (2015). Teoría del delito y juicio oral. Distrito Federal: Instituto de Investigaciones Juridicas UNAM.

Cámara de Diputados del H. Congreso de la Unión. (01 de 05 de 2020,01 de mayo). Constitución Política de los Estados Unidos Mexicanos. Ciudad de México, México: Diario Oficila de la Federación. Obtenido de http://www.diputados.gob.mx/LeyesBiblio/pdf/1_080520.pdf

Carrion , F. (Marzo de 2007). Ciudad segura. Programa de Estudios de la Ciudad. Percepción inseguridad ciudadana. Percepción inseguridad ciudadana. Quito, Ecuador, Ecuador: FLACSO sede Ecuador.

Carrión, F. (2005). La inseguridad ciudadana en América Latina. La inseguridad ciudadana en América Latina, 32.

Centro Internacional para la Prevención de la Criminalidad. (1995). Las estrategias de prevención del delito son las formas más viables de reducir. Montreal .

Centro internacional para la prevención de la criminalidad. (2010). Informe Internacional Sobre la Prevencion de la Criminalidad y la Seguridad Cotidiana: Tendencias y Perspectivas. Montréal.: Centro internacional para la prevención de la criminalidad.

Chalom, M., Léonard, L., Vanderschueren, F., \& Vézina, C. (2001). Seguridad ciudadana, participación social y buen gobierno: el papel de la policía. Santiago: Ediciones LOM.

Contreras, P., \& Montecinos, E. (2019). Democracia y participación ciudadana: Tipología y mecanismos para la implementación. Revista de Ciencias Sociales, 178-191.

Dammert, L. (2002). Participación comunitaria en la prevención del delito en América Latina. Chile.

De Cauter, F., \& Walgrave, L. (1999). Metodología de operación preventiva del proyecto. Acco.

Dijk, V. (1990). Crime Prevention Policy: Current State and Prospects. United States of America: National Institute of Justice. 
Estrada Rodriguez, J. (2013). Democracia, Estado y seguridad ciudadana. Alegatos, 367.

Fernández, C., \& Revilla, J. y. (2010). Emotions elicited by television violence. Scientific Journal of Media Literary, 36, 97-104.

Galindez Delvitto, M. (2013). Participación Ciudadana en la Prevención del Delito: ¿Estamos preparados? Buenos Aires, Argentina.

García Ramírez, S. (2002). Los desafíos de la seguridad pública en México. En En torno a la seguridad pública. Desarrollo penal y evolución del delito. México: Universidad Iberoamericana.

Garland, D. (2001). Crimen y Orden Social en la Sociedad Contemporanea. La cultura del control. Barcelona: Gedisa S.A.

Gilling, D. (1998). Crime Prevention: Theory, Policy and Politics. The British Journal of Criminology, 5.

Gomà, R. (2008). La acción comunitaria: transformación social y construcción de ciudadanía. Revista de Educación Social.

Graham, J., \& Trevor, B. (1995). Crime Prevention Strategies in Europe and North America. Londres: Criminal Justice Pr.

Guillen, A. K. (2009). Origen, espacio y niveles de participación ciudadana. Daena: International Journal of Good Conscience., 179-193.

Hobbes, T. (1651). Leviathan. Inglaterra: Andrew Crooke.

Jasso López, C. (2013). Percepción de inseguridad en México. Revista Mexicana de Opinión Pública, 12-29.

Julian, D., Reischl, T., \& Carrick, R. y. (2007). "Citizen participation-lessons from a local united way planning process". Journal of the American Planning Association, 345355.

Labra Díaz, C. (2011). El modelo de policía comunitaria: el caso chileno. Revista Chilena de Derecho y Ciencia Política, 51.

López Mejía, D. (2018). Introducción al Estudio del Derecho. Revista de Ciencia Júridica y Política, 101-112.

López Regonesi, E. (2000). Reflexiones acerca de la seguridad ciudadana en Chile: visiones y propuestas para el diseño de una política. Chile: Publicación de las Naciones Unidas .

Machicado, J. (2010). Concepto de Delito. Apuntes Jurídicos, 6.

Mangold, P. (1990). National Security and International Relations. New York: Taylor \& Francis Group, an informa business.

Marisol, S. (2003). La prevención en trabajo social,. La Habana: Editorial Félix Varela.

Merino, M. (2019). La participación ciudadana en la democracia. Ciudad de México: Instituto Nacional Electoral.

Montero, J. R. (1998). Actitudes hacia la Democracia en . Revista Española de Investigaciones Sociológicas España: legitimidad, descontento y desafección., 9-49.

Muñoz, F. (2018). Teoría General del Delito. Bogotá: Temis.

Ordóñez, J. (2019). Participación ciudadana y acción gubernamental: una curva de indiferencia para la seguridad pública en México. Espiral Estudios sobre Estado y Sociedad, 98.

Patiño Mariaca, D., \& Ruiz Gutiérrez, A. (2015). La justicia restaurativa: un modelo comunitarista de resolución de conflictos. Revista de la Facultad de Derecho y Ciencias Políticas, 218. 
Pegoraro, J. S. (2002). Las políticas de seguridad y la participación comunitaria en el marco de la violencia social. Buenos Aires: CLACSO, Consejo Latinoamericano de Ciencias Sociales.

Pérez, N., \& Núñez, G. (2008). La Participación Comunitaria en la Prevención del Delito: Experiencias Recientes en el Área Metropolitana de Caracas. Capítulo Criminológico Vol. 36, 112-113.

Real Academia Española. (27 de 05 de 2017). Real Academia Española. Obtenido de https://www.rae.es/

Real Academia Española. (2019). https://www.rae.es/. Obtenido de https://dle.rae.es/?w=prevención.

Rivera Ciriaco, R. V. (2018). Un modelo psicosocial para adolescentes con trayectorias delictivas de alto riesgo provenientes de Lima Metropolitana, Callao y Trujillo. 88.

Rodríguez, G., \& Montero, J. (2012). Modelos Preventivos del Delito.

Sanhueza, A. (2004). Participación Ciudadana en la Nueva Gestion Pública. Participa, 2.

Selmin, R. (2009). La prevención: estrategias, modelos y defi niciones en el contexto europeo. Urvio, Revista Latinoamericana de Seguridad Ciudadana, 49.

Sozzo, M. (2000). Seguridad Urbana y Tácticas de Prevención del Delito. Cuadernos de Jurisprudencia y Doctrina Penal, 104.

Torio, Á. (1984). Sección de los delitos por omisión. XIII Congreso Internacional de Derecho Penal, (pág. 694). El Cairo.

Turner, B. (1993). "Contemporary problems in the theory of citizenship”. Londres: Bryan S. Turner.

Unión, C. d. (2019, 27 de mayo). Ley General del Sistema Nacional de Seguridad Pública. Ciudad de México: Diario Oficial de la Federación. Obtenido de http://www.diputados.gob.mx/LeyesBiblio/pdf/LGSNSP_270519.pdf

Vega, E. (2017). El control y la prevención del delito como objeto de la criminología. Miscelánea Comillas. Revista de Ciencias Humanas y Sociales, 173.

Vilalta Perdomo, C. (2009). El miedo al crimen en México. Gestión y Política Pública, 8.

Waller, I. (1997). Prevención del Delito: La Nueva Esperanza de las Politicas de Urbanismo . En P. S. Europea. D.F: Siglo XXI.

Ziccardi, A. (2004). Introducción: Claves para el análisis de la participación ciudadana y las políticas sociales del espacio local. Instituto de Investigaciones Sociales, 9-19. 